\title{
Prevalence and prediction of calcification and plaques in radial artery grafts by ultrasound
}

\author{
Permyos Ruengsakulrach, MD, FRCST, a Mark Brooks, MB, BS, FRACR, ${ }^{\text {b }}$ Roger Sinclair, MB, BS, MD, FRCPA, \\ FRCPath, ${ }^{c}$ David Hare, MB, DPM, FRACP, ${ }^{d}$ Ian Gordon, MSc, PhD, ${ }^{\mathrm{e}}$ and Brian Buxton, MS, FRACS, ${ }^{a}$ \\ Melbourne, Victoria, Australia
}

$\mathrm{T}$ he radial artery (RA) is increasingly being used as a conduit for coronary artery bypass grafting and has shown promising midterm graft patency rates. ${ }^{1,2}$ A previous study of ours indicated that, on the basis of histopathologic examination, the RA has a higher incidence of pre-existing intimal hyperplasia, medial calcification, and atherosclerosis than the internal thoracic artery. ${ }^{3}$ However, the findings of this study were limited by the fact that we examined only the distal end of the RA. Furthermore, the RAs of patients found to have severe calcification during intraoperative exploration were not harvested and therefore were not subjected to histopathologic study.

In this study we used ultrasound to examine the entire length of the RA for plaques and calcification preoperatively. Our aims were (1) to investigate the prevalence of RA plaques, calcification, and stenoses and (2) to identify patients with a high risk of having RA plaques and calcification.

\section{Patient Population}

Seventy-three patients scheduled for coronary artery bypass grafting between July 1998 and May 1999 entered this study. The ultrasound study was performed on the day before the operation. There was no selection of patients in relation to patient characteristics, other than the timing of their operation. The 9 potential risk factors for RA plaques and calcification are listed in Table 1.

\section{Ultrasound Examination ( $N=73$ Arms)}

Ultrasound examination was performed the day before the operation by one of two sonographers using an ATL (Advanced

From the Departments of Cardiac Surgery, ${ }^{a}$ Radiology, ${ }^{\mathrm{b}}$ Pathology, ${ }^{\mathrm{c}}$ and Cardiology, ${ }^{\mathrm{d}}$ Austin \& Repatriation Medical Centre, Heidelberg, and the Department of Mathematics and Statistics, ${ }^{e}$ the University of Melbourne, Melbourne, Victoria, Australia.

Received for publication Aug 8, 2000; accepted for publication Dec 20, 2000.

Address for reprints: Professor Brian F. Buxton, Department of Cardiac Surgery, Austin Campus, HSB-5, Austin \& Repatriation Medical Centre, Studley Rd, Heidelberg, Victoria 3084, Australia (E-mail: brian.buxton@ armc.org.au).

J Thorac Cardiovasc Surg 2001;122:398-9

Copyright $\odot 2001$ by The American Association for Thoracic Surgery

$0022-5223 / 2001 \$ 35.00+0 \quad \mathbf{1 2 / 5 4 / 1 1 4 0 9 6}$

doi:10.1067/mtc.2001.114096
Technology Laboratories, Bothell, Wash), HDI (High Definition Imaging) 5000 system with a $10-\mathrm{MHz}$ compact linear array transducer. The entire length of the RA was examined for calcification and echogenic plaques. Echogenic foci in the arterial wall with posterior acoustic shadowing were recorded as calcification. Those without posterior acoustic shadowing were recorded as echogenic plaques. Stenosis was defined as a focal increase in peak systolic velocity compared with the proximal arterial segment.

\section{Statistical Analysis}

Nine clinical risk factors (Table 1) for RA plaques and calcification were included in logistic regression analyses as independent variables. A forward stepwise procedure was used to identify a suitable model. Exact logistic regression was used to fit the model to the data after the model was identified. ${ }^{4}$ Positive RA calcification and any RA abnormality (RA calcification or echogenic plaques) by ultrasound were included as dependent variables. The presence of plaques was not analyzed as a dependent variable because only 5 of 73 patients had plaques. $P$ values and $95 \%$ confidence intervals were reported for key results. ${ }^{5}$

\section{Results}

RA calcification (intimal or medial calcification) was detected by ultrasound in $24.7 \%$ (18/73) of RAs. Echogenic plaques were found in $6.8 \%$ (5/73). The overall incidence of RA abnormality (calcification or echogenic plaques) was $31.5 \%$. No stenoses were detected.

The forward stepwise logistic procedure selected a model with the clinical risk factors of age, male sex, and carotid artery disease for RA calcification alone. Since 2 of these factors were of borderline significance, the logistic regression model involving all 3 factors was fitted to the data by means of an exact procedure (age: $P=.03$; sex: $P=.08$; carotid disease, $P=.02$ ).

The stepwise logistic regression procedure identified carotid disease and peripheral vascular disease as suitable risk factors for any ultrasound-detected RA disease (calcification or plaque). With the use of these 2 variables, a logistic regression was fitted to the data by means of an exact procedure; the results are shown in Table 2.

\section{Discussion}

The prevalence of RA calcification and echogenic plaques as detected by ultrasound was $25 \%$ and $7 \%$, respectively. These figures were higher than those in a previous study of ours in which only the distal portion of the RA was examined histopathologically. ${ }^{3}$ This difference probably reflects an underestimation of disease in our previous study because of limited tissue sampling. 
TABLE 1. Clinical risk factors $(\mathbf{n}=\mathbf{7 3})$

\begin{tabular}{lc}
\hline Age (y) & \\
Range & $40-85$ \\
Mean \pm SD & $67.1 \pm 9.8$ \\
Sex & $60(82.2 \%)$ \\
Male & $13(17.8 \%)$ \\
Female & \\
Smoking history & $16(21.9 \%)$ \\
$\quad$ Never smoked & $50(68.5 \%)$ \\
Previous & $7(9.6 \%)$ \\
Current & \\
Diabetes & $51(69.9 \%)$ \\
$\quad$ None & $3(4.1 \%)$ \\
Diet control & $13(17.8 \%)$ \\
Oral hypoglycemic drugs & $6(8.2 \%)$ \\
Insulin injection & $43(58.9 \%)$ \\
Hypertension & $19(26.0 \%)$ \\
Carotid disease & $23(31.5 \%)$ \\
Peripheral vascular disease & $11(10 \%)$ \\
Cerebrovascular disease & $53(48.2 \%)$ \\
Hypercholesterolemia &
\end{tabular}

The standard practice for determining whether a vessel is suitable for use as a bypass graft is to examine the RA visually and by palpation during the operation. Usually RAs with gross calcification are excluded. Such evaluation is subjective and is likely to underestimate the degree of arterial disease present. In contrast, ultrasound is a noninvasive procedure that can be performed preoperatively.

A potential weakness of ultrasound is that it is unable to differentiate between medial calcification (Mönckeberg's medial sclerosis) and calcification in the intima. Theoretically, it is important to distinguish between these two types of calcification because intimal calcification is related to atherosclerosis and often leads to luminal compromise. In contrast, the effect of medial calcification on graft patency remains unknown. Further improvement in ultrasound imaging may enable us to distinguish medial calcification from calcified intimal plaque.

Age, male sex, and the presence of carotid artery disease predicted RA calcification as detected by ultrasound, whereas the risk factors associated with any RA disease were carotid disease and peripheral vascular disease. Note that some of these associations are of borderline statistical significance (see Table 2), but these associations involve quite large odds ratios. The estimation of effects, however, is imprecise because of the relatively small size of the study and the wide confidence intervals.
TABLE 2. Results of exact logistic regression analysis of dependent variables for RA calcification and echogenic plaques

\begin{tabular}{lccc}
\hline & Pvalue & Odds ratio & 95\% CI \\
\hline Peripheral vascular disease & .05 & 3.6 & $1.0-13$ \\
Carotid disease & .08 & 3.3 & $0.9-13$ \\
\hline
\end{tabular}

$\mathrm{Cl}$, Confidence intervals.

Is it worth screening the RA in all patients before RA harvesting? In our opinion, RA screening by ultrasound improves the graft selection process and may potentially improve graft patency by avoiding the use of diseased arteries. An immediate advantage to patients is the avoidance of unnecessary forearm surgery. Another benefit of ultrasound is that it can also be used to preoperatively assess the collateral circulation of the hand. It is also useful for identifying anatomic variations (eg, bifurcation and diameter of the RA), as well as previous traumatic injury to the RA due to, for instance, previous catheterization. Furthermore, the procedure is quick, easy to perform, and noninvasive. Therefore, we recommend performing RA screening in all patients if possible, but especially in those with carotid and peripheral vascular disease.

To our knowledge, this is the first study to document the prevalence of RA calcification as determined by ultrasound and to identify the risk factors for RA calcification and abnormalities. Our findings suggest that the prevalence of RA calcification and plaques is relatively high in patients needing coronary artery bypass grafting. We found that older or male patients or those with carotid artery disease are at a high risk for RA calcification alone; those who have carotid disease or peripheral vascular disease tend to have a higher risk of any RA abnormality. Care should be taken when using the RA in such patients.

\section{References}

1. Acar C, Ramsheyi A, Pagny JY, Jebara V, Barrier P, Fabiani JN, et al. The radial artery for coronary artery bypass grafting: clinical and angiographic results at five years. $J$ Thorac Cardiovasc Surg. 1998;116:981-9.

2. Possati G, Gaudino M, Alessandrini F, Luciani N, Glieca F, Trani C, et al. Midterm clinical and angiographic results of radial artery grafts used for myocardial revascularization. J Thorac Cardiovasc Surg. 1998;116:1015-21.

3. Ruengsakulrach P, Sinclair R, Komeda M, Raman J, Gordon I, Buxton B. Comparative histopathology of the radial artery versus the internal thoracic artery and risk factors for development of intimal hyperplasia and atherosclerosis. Circulation. 1999;100(Suppl):II-139-44.

4. Mehta C, Patel N. LogXact for windows. Cambridge (MA): Cytel; 1996.

5. Gardner MJ, Altman DG. Confidence intervals rather than $\boldsymbol{P}$ values: estimation rather than hypothesis testing. BMJ. 1986;292:746-50. 